\title{
The Measurement of Orthopaedic Surgeon Burnout Using a Validated Wearable Device
}

\author{
Kyle R. Sochacki, M.D., David Dong, B.S., Leif Peterson, Ph.D., Patrick C. McCulloch, M.D., \\ and Joshua D. Harris, M.D.
}

\begin{abstract}
Purpose: The purpose of this study was (1) to determine the prevalence of burnout in orthopaedic surgeons and (2) to determine whether there is an association or correlation between subject-specific variables (age, attending physician, resident, postgraduate year level, gender, number of calls, total hours worked, and total hours of sleep) and burnout. Methods: Surgeons were prospectively enrolled and provided with a validated wearable device. Subject-specific variables were recorded. Participants completed the Maslach Burnout Inventory and Patient-Reported Outcomes Measurement Information System (PROMIS-29) weekly. Burnout and burnout risk were defined. Multivariate analysis and bivariate correlations were used to determine the association and correlation between subject-specific variables and burnout. Residents were compared to attending surgeons. Results: Of the 26 enrolled subjects, 21 (15 males, 6 females; mean age $37.2 \pm 10.9)$ completed the 4 -week study. Residents worked significantly more hours per week than attending surgeons $(68.5 \pm 15.2$ versus $49.9 \pm 7.5, P=0.009)$. Of the orthopaedic surgeons, $6(28.6 \%)$ experienced burnout, and $7(33.3 \%)$ orthopaedic surgeons were at risk for burnout. There was no significant difference in burnout rates between residents and attending surgeons $(P>0.05)$. The number of overnight calls was significantly correlated with increased burnout $(\mathrm{r}=0.435, P=0.049)$. Female gender was significantly associated $(P=0.041)$ and correlated $(\mathrm{r}=0.558, P=0.009)$ with burnout. There was no significant association with burnout between the number of hours worked and hours of sleep. Conclusions: The rate of burnout was less than $50 \%$ among orthopaedic surgeons. The number of overnight calls and female gender are significantly correlated with increased burnout. There was no significant correlation between hours worked and hours of sleep in surgeon burnout. Clinical Relevance: Burnout is an increasingly common problem among orthopaedic surgeons, and it can have significant negative effects on surgeons' health and patients' outcomes. Identifying the predictors of burnout would allow surgeons to address these risk factors and reduce burnout.
\end{abstract}

\section{Introduction}

B urnout is a syndrome that results from high levels of work stress characterized by emotional exhaustion, depersonalization and lack of feelings of personal accomplishment. ${ }^{1-3}$ Work overload, lack of control, insufficient rewards, lack of a sense of community, and lack of fairness are factors that can lead to

From the Houston Methodist Orthopedic and Sports Medicine, 6445 Main Street, Suite 2500, Houston, Texas 77030.

The authors report that they have no conflicts of interest in the authorship and publication of this article. Full ICMJE author disclosure forms are available for this article online, as supplementary material.

Received June 18, 2019; accepted September 7, 2019.

Address correspondence to Joshua D Harris, Houston Methodist Orthopedic and Sports Medicine, 6445 Main Street, Suite 2500, Houston, Texas 77030, U.S.A.E-mail: joshuaharrismd@gmail.com

(C) 2019 by the Arthroscopy Association of North America. Published by Elsevier Inc. This is an open access article under the CC BY-NC-ND license (http://creativecommons.org/licenses/by-nc-nd/4.0/).

2666-061X/19774

https://doi.org/10.1016/j.asmr.2019.09.004 burnout. ${ }^{1,3}$ As such, this syndrome has a high prevalence in health care workers, and students, nurses, residents, and attending physicians are at higher risk than the general population. ${ }^{3-5}$

Several studies of surgeons and nonsurgeons have found that burnout can occur at a very high rate and is increasing in prevalence; $37.9 \%$ of physicians reported burnout in 2011 , and $46.9 \%$ of physicians experienced burnout in $2014 .^{6-9}$ The prevalence of burnout in orthopaedic surgeons is even higher. Reported burnout rates are as high as $65 \% .{ }^{10-13}$ This can lead to significant health risks for surgeons: increased depression, diabetes mellitus, heart disease, metabolic syndrome, myocardial infarction, and suicidal ideation. ${ }^{9,13-15}$ Burnout can also have a negative effects on patient care, resulting in decreased performance by surgeons and increased rates of medical errors. ${ }^{16,17}$

The Maslach Burnout Inventory (MBI) is a validated 22-item questionnaire that is considered the goldstandard tool for measuring burnout. ${ }^{3}$ Previous studies have demonstrated high levels of emotional 
exhaustion or depersonalization (2 of the 3 MBI dimensions) as the foundation of burnout in physicians. ${ }^{5,6,18,19}$ There also appears to be a physiologic component to physician burnout and decreased performance that is associated with increased number of hours worked, number of calls and sleep deprivation (fewer than 7 hours of sleep per night). ${ }^{6,20-22}$ However, prior studies of orthopaedic surgeons are limited to subjective reporting of sleep hours and are without objective measurements. Additionally, there have been few burnout studies in orthopaedic surgery residents and attending surgeons since the 2003 and 2011 Accreditation Council for Graduate Medical Education work-hour restrictions commenced. ${ }^{13,23-25}$ Given the incomplete understanding of sleep patterns and burnout following the work-hour restrictions and the increasing interest in physician and patient safety, it is important to understand the effects of sleep on orthopaedic surgeon burnout. ${ }^{13,26}$

The purposes of this study were (1) to determine the prevalence of burnout in orthopaedic surgeons and (2) to determine whether there is an association or correlation between subject-specific variables (age, attending physician, resident, postgraduate year [PGY] level, gender, number of calls, total hours worked, and total hours of sleep) and burnout. The authors hypothesized that (1) there will be less than $50 \%$ of subjects with burnout and (2) there would be a strong positive association and correlation between increased number of calls, increased hours worked, and decreased hours of sleep and increased burnout, with no association or correlation between age, attending physician, resident, PGY level, and gender and increased burnout.

\section{Methods}

Institutional review board approval was obtained. The study assessed 28 orthopaedic surgery professionals, all from a single academic institution; residents $(\mathrm{n}=14)$ and attending surgeons $(\mathrm{n}=14)$ were assessed for study eligibility between October 2017 and November 2017 (Fig. 1). Those participating in the study included 1 PGY-5, 3 PGY-4s, 2 PGY-3s, 3 PGY-2s, and 2 PGY-1s. Of the attending surgeons, 4 were fellowship-trained in orthopaedic sports medicine, 2 were fellowship-trained in hand and upper-extremity surgery, 1 was fellowshiptrained in adult reconstruction, 1 was fellowshiptrained in foot and ankle surgery, and 1 was dual fellowship-trained in spine and oncology. The mean time in practice for attending surgeons was $14.1 \pm 9.4$ years. All subjects signed informed consents prior to entering the study. Subjects were eligible for inclusion if they were certified by the American Board of Orthopaedic Surgery as attending surgeons or orthopaedic surgery residents from a single academic institution. Subjects were excluded from eligibility if they were not certified by the American Board of Orthopaedic Surgery (attending physicians), not currently a PGY-1 to PGY-5 orthopaedic surgery resident or had not completed the minimum compliance of 4 weeks. The authors were blinded to the individual levels' statistics.

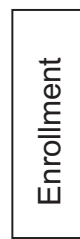

\begin{tabular}{|l|l|}
\hline $\begin{array}{l}\text { Number of subjects } \\
\text { assessed for eligibility } \\
(n=28)\end{array}$ & $\longrightarrow \begin{array}{l}\text { Number of subjects } \\
\text { declined participation } \\
(n=2)\end{array}$ \\
\hline
\end{tabular}

Number ofsubjects allocated WHOOP $(n=26)$
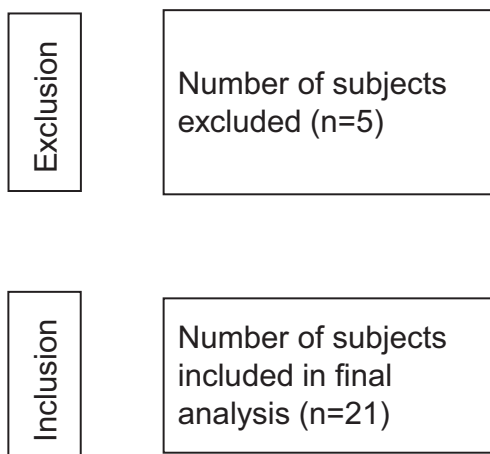

Fig. 1. Flow diagram of patient exclusion and inclusion. 
All participants were provided with a WHOOP (WHOOP, Boston, MA) wearable device, to be worn at all times during the 4-week study period, that measured data continuously. The WHOOP is a validated device that uses photoplethysmography to measure 5 metrics: heart rate, heart rate variability, ambient temperature, motion and movement, and skin response to measure and analyze a persons' sleep. ${ }^{27}$ Sleep was recorded as total sleep based on the WHOOP data. Total sleep was analyzed as mean hours slept per day per week. Sleep deprivation was defined as getting fewer than 7 hours of sleep per day. ${ }^{22}$

Demographic information such as age, gender (male/ female) and PGY level (resident) were recorded for all subjects at the beginning of the study. All participants recorded the number of days on call per week at the end of each week. All participants subjectively reported the number of hours worked per day at the end of each week. Work hours were defined as the number of hours spent in the outpatient or inpatient setting participating in patient care. Participants completed the MBI-Human Services Survey and Patient-Reported Outcomes Measurement Information System (PROMIS)-29 version 2.1 weekly for the duration of the study period.

The MBI is categorized into emotional exhaustion, depersonalization and personal achievement. Burnout was classified as low ( $\leq 17$ for emotional exhaustion, $\leq 5$ for depersonalization, $\geq 40$ for personal achievement); moderate (18-29 for emotional exhaustion, 6-11 for depersonalization, 34-39 for personal achievement); or high ( $\geq 30$ for emotional exhaustion, $\geq 12$ for depersonalization, $\leq 33$ for personal achievement). ${ }^{3}$ A subject was considered to have burnout if he or she had a high (i.e. $\geq 30$ for emotional exhaustion, $\geq$ 12 for depersonalization, $\leq 33$ for personal achievement) score in at least 1 of the 3 MBI domains, and a subject was considered to be at risk for burnout if he or she had a moderate score in at least 1 of the $3 \mathrm{MBI}$ domains. $3,28,29$

The PROMIS-29 is categorized into 8 subdomains: (1) physical function; (2) anxiety; (3) depression; (4) fatigue; (5) sleep disturbance; (6) ability to participate in social roles and activities; (7) pain interference; and (8) pain intensity. Each PROMIS-29 domain is scored from 0 -100; scores $>50$ are considered worse than those in the general population for all categories except physical function $(<50$ is worse than that in the general population); ability to participate in social roles and activities ( $<50$ is worse than that in general population); and pain intensity (scored 0-10, with higher scores being worse). ${ }^{30,31}$

Data analysis was performed using SPSS Statistics software, version 20 (IBM, Armonk, NY). The significance of differences in means of continuous variables between 2 groups was determined by the Student $t$ test.
The differences in categorical variables between groups were determined by the $\chi^{2}$ test. Bivariate correlations were determined by Spearman correlation coefficients. Correlation was defined as a perfect negative linear relationship $\left(\mathrm{r}, \mathrm{r}_{\mathrm{s}}=-\mathrm{l}\right.$ :), strong negative linear relationship $\left(\mathrm{r}, \mathrm{r}_{\mathrm{s}}=-0.70\right)$, moderate negative relationship $\left(\mathrm{r}, \mathrm{r}_{\mathrm{s}}=-0.50\right)$, weak negative linear relationship $(\mathrm{r}$, $\left.r_{\mathrm{s}}=-0.30\right)$, no linear relationship $\left(\mathrm{r}, \mathrm{r}_{\mathrm{s}}=0\right)$, weak positive linear relationship $\left(r, r_{s}=+0.30\right)$, moderate positive relationship $\left(r, r_{s}=+0.50\right)$, strong positive linear relationship $\left(r, r_{s}=+0.70\right)$, or perfect positive linear relationship $\left(r, r_{s}=+1\right)$. Residents were compared to attending surgeons. Multivariate linear regression models were built to determine the effects of independent variables (age, attending physician, resident, PGY level, gender, number of calls, total hours worked, and total hours of sleep) on orthopaedic surgeon burnout (MBI and PROMIS-29). Categorical variables were coded as dummy variables (i.e. for gender, 0 represented male and 1 represented female). All $P$ values were reported with significance set at $P<0.05$.

\section{Results}

Of the 28 subjects assessed for eligibility, 2 subjects declined to participate, and 5 subjects did not complete the minimum WHOOP compliance ( 4 weeks) and were excluded from analysis. Of the $26(80.8 \%)$ enrolled, 21 (12 residents and 9 attending surgeons) completed the minimum 4-week duration of the study and were included in the final analysis.

Subjects' demographics are shown in Table 1. Orthopaedic surgeons obtained $6.5 \pm 0.8$ hours of sleep per night, on average. Of the 21 orthopaedic surgeons, $14(67.7 \%)$ slept fewer than the recommended 7 hours per night. Residents also worked significantly more hours per week than attending surgeons $(68.5 \pm 15.2$ vs $49.9 \pm 7.5, P=0.009$ ). There were no other significant differences in demographics between resident and attending surgeons $(P>0.05)$.

Six $(28.6 \%)$ orthopaedic surgeons $(33.3 \%$ of residents and $22.2 \%$ of attending surgeons) experienced burnout, and 7 (33.3\%) orthopaedic surgeons ( $25.0 \%$ of residents and $44.4 \%$ of attending surgeons) were at risk for burnout. All subjects with burnout or at risk for burnout scored high or moderate, respectively, on the depersonalization domain of the MBI (Table 2). There was no significant difference in burnout rates between residents and attending surgeons $(P>0.05)$. Of the subjects, $19(90.5 \%)$ had low, and $2(9.5 \%)$ had moderate levels of emotional exhaustion. Eight $(38.1 \%), 7(33.3 \%)$ and $6(28.6 \%)$ orthopaedic surgeons had low, moderate or high levels of depersonalization, respectively. Twenty $(95.2 \%)$ and $1(4.8 \%)$ had high and moderate levels of personal achievement, respectively. 
Table 1. Subject Demographics

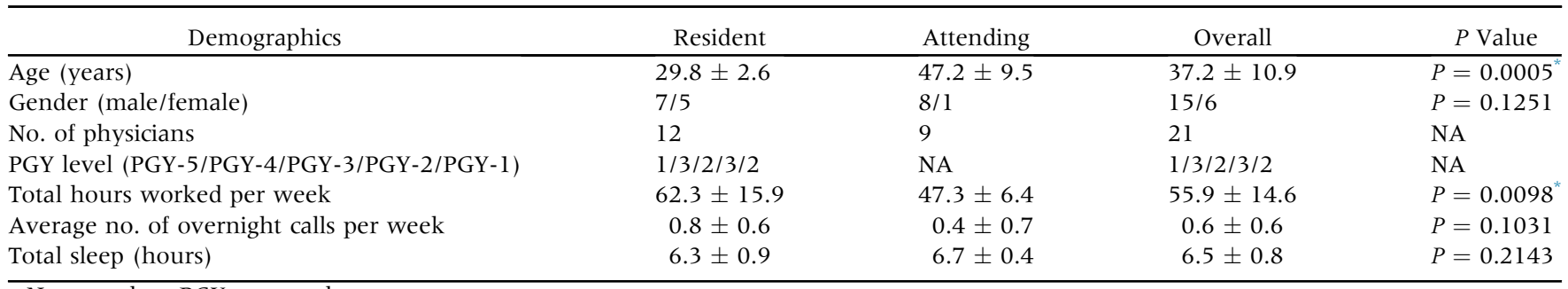

No., number; PGY, postgraduate year.

*Statistically significant $(P<.05)$.

A number of study subjects scored worse than the general population on the following PROMIS-29 subscores: $5(23.8 \%)$ for physical function, $5(23.8 \%)$ for anxiety, $4(19.0 \%)$ for depression, $9(42.9 \%)$ for fatigue, $8(38.1 \%)$ for sleep disturbance, $11(52.4 \%)$ for ability to participate in social roles and activities, and 3 $(14.3 \%)$ for pain interference (Table 2). Residents had significantly worse depression than attending surgeons $(45.8 \pm 5.0$ vs $41.9 \pm 1.5, P=0.035)$. There were no other significant PROMIS score differences between resident and attending surgeons (Table 2).

In multivariate models (Table 3), female gender had the strongest association with burnout based on the depersonalization score $(P=0.041)$. Female gender also had the strongest association with depression $(P=0.004)$ and worse physical function $(P=0.008)$ based on the PROMIS-29 scores. The number of overnight calls was strongly associated with worse physical function $(P=0.008)$, depression $(P=0.010)$ and fatigue $(P=0.014)$ on the PROMIS-29 score. Greater age was strongly associated with increased pain on the PROMIS-29 score $(P=0.002)$. There was no significant association between hours worked and hours of sleep with surgeon burnout $(P>0.05)$.

In bivariate correlation analysis, the number of overnight calls had a weak positive correlation with burnout, as scored by emotional exhaustion $(r=0.435)$ and a moderate negative correlation with physical functioning ( $\mathrm{r}=0-0.638$ ) (Table 4). PGY level had a moderate negative correlation with burnout as scored by depersonalization $(\mathrm{r}=-0.652)$, a moderate negative correlation with anxiety $(\mathrm{r}=-0.597)$ and a strong negative association with pain intensity $(\mathrm{r}=-0.71 \mathrm{l})$. Female gender had a moderate positive correlation with depersonalization $(\mathrm{r}=0.558)$, a moderate negative correlation with physical functioning $(\mathrm{r}=-0.652)$ and a moderate positive correlation with depression $(\mathrm{r}=0.640)$ (Table 4). There was no significant correlation between hours worked and hours of sleep with surgeon burnout.

\section{Discussion}

The study hypotheses were partially confirmed; $28.6 \% \%$ of orthopaedic surgeons had burnout, and an additional $33.3 \%$ were at risk for burnout. The number of overnight calls was significantly correlated with increased burnout, anxiety, depression, fatigue, and worse physical functioning, and female gender was significantly correlated with burnout, depression and worse physical function. However, there was no significant association between hours worked and hours of sleep with orthopaedic surgeon burnout.

Burnout is a syndrome that consists of emotional exhaustion, depersonalization and lack of personal accomplishment. ${ }^{1-3}$ Previous studies have shown that, compared to other occupations, physicians experience

Table 2. Orthopaedic Surgeon Burnout

\begin{tabular}{|c|c|c|c|c|}
\hline Burnout Statistic & Resident & Attending & Overall & $P$ Value \\
\hline MBI: Emotional exhaustion & $9.2 \pm 9.2$ & $8.8 \pm 5.1$ & $9.1 \pm 7.5$ & $P=0.917$ \\
\hline MBI: Depersonalization & $12.5 \pm 13.2$ & $7.3 \pm 6.0$ & $10.3 \pm 10.8$ & $\mathrm{P}=0.285$ \\
\hline MBI: Personal achievement & $42.7 \pm 6.7$ & $44.4 \pm 3.5$ & $43.5 \pm 5.5$ & $P=0.514$ \\
\hline PROMIS: Anxiety & $46.8 \pm 6.8$ & $43.9 \pm 5.6$ & $45.6 \pm 6.4$ & $P=0.302$ \\
\hline PROMIS: Depression & $45.8 \pm 5.0$ & $41.9 \pm 1.5$ & $44.1 \pm 4.3$ & $P=0.035^{*}$ \\
\hline PROMIS: Fatigue & $50.2 \pm 10.8$ & $47.4 \pm 9.2$ & $48.9 \pm 9.9$ & $P=0.545$ \\
\hline PROMIS: Pain interference & $39.5 \pm 13.0$ & $44.2 \pm 4.6$ & $41.5 \pm 10.4$ & $P=0.312$ \\
\hline PROMIS: Pain intensity & $0.41 \pm 0.83$ & $0.75 \pm 1.2$ & $0.56 \pm 1.0$ & $P=0.470$ \\
\hline
\end{tabular}

MBI, Maslach Burnout Inventory; PROMIS-29, Patient-Reported Outcomes Measurement Information System-29.

*Statistically significant $(P<.05)$. 
Table 3. Multivariate Analysis of the Effect of Subject Factors on Orthopaedic Surgeon Burnout

\begin{tabular}{|c|c|c|}
\hline Variable & Regression Coefficient & $P$ Value \\
\hline \multicolumn{3}{|l|}{ MBI: Depersonalization } \\
\hline Female gender & 2.214 & $P=0.041^{*}$ \\
\hline \multicolumn{3}{|l|}{ PROMIS: Physical function } \\
\hline No. of overnight calls & -3.149 & $P=0.008^{*}$ \\
\hline Female gender & -3.329 & $P=0.005^{*}$ \\
\hline \multicolumn{3}{|l|}{ PROMIS: Anxiety } \\
\hline No. of overnight calls & 2.953 & $P=0.018^{*}$ \\
\hline \multicolumn{3}{|l|}{ PROMIS: Depression } \\
\hline No. of overnight calls & 3.690 & $P=0.010^{*}$ \\
\hline Female gender & 4.467 & $P=0.004^{*}$ \\
\hline \multicolumn{3}{|l|}{ PROMIS: Fatigue } \\
\hline No. of overnight calls & 2.725 & $P=0.014^{*}$ \\
\hline \multicolumn{3}{|l|}{ PROMIS: Pain } \\
\hline Age & 3.550 & $P=0.002^{*}$ \\
\hline
\end{tabular}

significantly higher burnout rates; burnout rates for orthopaedic surgeons are as high as 65\%.,10-13,17 High surgeon burnout rates pose significant health and safety risks for surgeons, including increased depression, diabetes mellitus, heart disease, metabolic syndrome, and myocardial infarction. ${ }^{9,13,14}$ Burnout can also have negative effects on patient care, resulting in decreased surgeon performance and increased rates of medical errors. ${ }^{16,17}$ Because of the detrimental effects of burnout on surgeon and patient health, the Accreditation Council for Graduate Medical Education restricted resident work hours in 2003 and 2011..$^{24,25}$ Since the adoption of the new hour restrictions, several retrospective studies have demonstrated conflicting results regarding improved patient safety and resident welfare. ${ }^{32-37}$

Table 4. Bivariate Correlation of Subject Factors and Orthopaedic Surgeon Burnout

\begin{tabular}{lcc}
\hline \multicolumn{1}{c}{ Variable } & Correlation Coefficient & $P$ Value \\
\hline $\begin{array}{lll}\text { MBI: emotional exhaustion } \\
\text { No. of overnight calls }\end{array}$ & 0.435 & $P=0.049^{*}$ \\
MBI: epersonalization & -0.652 & $P=0.021^{*}$ \\
$\quad$ PGY level & 0.558 & $P=0.009^{*}$ \\
$\quad$ Female gender & & \\
PROMIS: physical function & -0.638 & $P=0.002^{*}$ \\
$\quad$ No. of overnight calls & -0.652 & $P=0.001^{*}$ \\
$\quad$ Female gender & -0.597 & $P=0.040^{*}$ \\
PROMIS: anxiety & & \\
$\quad$ PGY level & 0.640 & $P=0.002^{*}$ \\
PROMIS: depression & & \\
$\quad$ Female gender & -0.711 & $P=0.014^{*}$ \\
PROMIS: pain intensity & &
\end{tabular}

MBI, Maslach Burnout Inventory; No., number; PGY, postgraduate year; PROMIS, Patient-Reported Outcomes Measurement Information System.

${ }^{*}$ Statistically significant $(P<0.05)$.
The current study demonstrated that $28.6 \%$ of orthopaedic surgeons $(33.3 \%$ of residents and $22.2 \%$ of attending surgeons) experienced burnout, and $33.3 \%(25.0 \%$ of residents and $44.4 \%$ of attending surgeons) were at risk of burnout. This burnout rate is significantly lower than that shown in prior studies of orthopaedic surgeons, which showed significantly worse scores for emotional exhaustion, depersonalization and personal achievement compared to the current study. ${ }^{10-13,23}$ Those studies were conducted prior to the implementation of the current work-hour restrictions; at the time, average work hours for orthopaedic surgeons amounted to as many as 88 hours per week compared to 56 hours per week in the current study. It is conceivable that the work-hour restrictions have been able to reduce the risk of burnout in orthopaedic surgery residents.

Additionally, the studies by Sargent et al. found a positive correlation between increased work hours and depersonalization for residents and faculty. ${ }^{13,23}$ This differs from the current study, in which total hours worked were not significantly associated or correlated with increased burnout.

However, the present study did find that the number of calls was significantly correlated with increased burnout. Balch et al. found similar results in general surgeons. ${ }^{38}$ This leads to the conclusion that the quality (overnight vs daytime) of the work hours may be more important than the quantity (total hours worked) with regard to orthopaedic surgeon burnout. The number of calls was also significantly associated with increased anxiety, depression, fatigue, and worse physical functioning on the PROMIS-29 score, further strengthening the argument.

Orthopaedic surgeons in the current study slept an average of 6.5 hours per night, and $67.7 \%$ slept fewer than the recommended 7 hours of sleep per night, indicating sleep deprivation. ${ }^{22}$ Interestingly, hours of sleep were not significantly associated or correlated with surgeon burnout in the present study. This differs from a prior study by Sargent et al., which found there to be a significant correlation between sleep deprivation and all measures of physician burnout. ${ }^{13}$ The discrepancy is likely to be due to the present study having used a validated wearable device to objectively quantify the total amount of sleep per night as compared to using a subjective scale of sleep deprivation. As one would expect, experiencing burnout would likely lead to feeling more sleep deprived, regardless of the actual hours of sleep obtained each night.

Female gender was also significantly associated or correlated with burnout, depression and worse physical function. This is not unexpected; previous studies have demonstrated that female surgeons are more likely to feel fatigued or burned out. ${ }^{39}$ Additionally, there is a reported higher rate of dissatisfaction with work-life 
balance in female surgeons despite working similar hours and taking a similar number of overnight calls compared to their male counterparts. ${ }^{40}$ These findings are likely to be due to females' in medicine having higher rates of psychological distress and depression, which have been shown to increase burnout rates. ${ }^{39,40}$

\section{Limitations}

There are limitations to this study. It was limited to orthopaedic surgery residents and attending physicians in a single academic institution, leading to a smaller sample size and selection bias. However, the authors tried to limit the bias through strict inclusion and exclusion criteria with $80.8 \%$ follow-up. The conclusions, therefore, may not be generalizable to all orthopaedic surgeons and physicians in other subspecialties from different regions because residents and attending surgeons from larger academic institutions tend to experience greater levels of burnout. ${ }^{13}$ It is also possible that the surgeons' responses to the MBI and PROMIS29 were not entirely accurate. Distrust of surveys, fear of retribution or concerns for loss of confidentiality can affect the responses in medical education research. ${ }^{41}$ Additionally, we conducted the study for 4 weeks. Therefore, we were probably unable to determine the effects of changes in patient volume, clinic, operating room, rotation (resident), call schedules, and sleep quantity on surgeon burnout over an extended period of time. Inclusion of multiple residents from different rotations and PGY levels as well as attending physicians from different subspecialties attempted to mitigate this. Hours worked were subjectively recorded at the end of each week. Although this eased data collection and increased response rates compared to daily recording, it is subject to recall bias and may lead to over- or underestimation of total hours worked. In order to decrease the subjectivity of sleep quantity seen in previous studies, the WHOOP device was used. Sleep polysomnography is the gold standard for objectively evaluating sleep. However, it is not well suited for longterm monitoring in multiple subjects. As such, the current sleep data are based on photoplethysmography, which has been validated against polysomnography and has shown moderate agreement. ${ }^{27}$ Despite validation, the objective sleep measures may differ from data obtained using sleep polysomnography.

\section{Conclusions}

The rate of burnout was less than $50 \%$ among orthopaedic surgeons. The number of overnight calls and female gender are significantly correlated with increased burnout. There was no significant correlation between hours worked and hours of sleep with surgeon burnout.

All the authors have been actively involved in the planning and enactment of the study and have assisted with the preparation of the submitted article. The manuscript has been read and approved by all authors.

The authors confirm that this is original work and has not been submitted, presented or published elsewhere.

Patrick C. McCulloch has the following disclosures: speaker's bureau/paid presentation by company/supplier (Vericel); research support from company/supplier (DePuy, A. Johnson \& Johnson Company; Arthrex); Medical/Orthopaedic Publications editorial/governing board (Journal of Knee Surgery; Orthobullets.com). Joshua D. Harris has the following disclosures: AAOS: board or committee member; American Orthopaedic Society for Sports Medicine: Board or committee member; Arthroscopy: editorial or governing board; Arthroscopy Association of North America: Board or committee member; DePuy, A. Johnson \& Johnson Company: research support; Frontiers In Surgery: editorial or governing board; NIA Magellan: paid consultant; SLACK Incorporated: publishing royalties, financial or material support; Smith $\delta$ Nephew: paid presenter or speaker, paid consultant, research support; Ossur: paid speaker. Kyle R Sochacki, David Dong and Leif Peterson declare no potential conflicts of interest with respect to the research, authorship and/or publication of this article.

\section{References}

1. Maslach C. What have we learned about burnout and health? Psychol Health 2001;16:607-611.

2. Maslach C, Leiter MP. New insights into burnout and health care:Strategies for improving civility and alleviating burnout. Med Teach 2017;39:160-163.

3. Maslach C, Schaufeli WB, Leiter MP. Job burnout. Annu Rev Psychol 2001;52:397-422.

4. Dimou FM, Eckelbarger D, Riall TS. Surgeon burnout: A systematic review. J Am Coll Sur 2016;222:1230-1239.

5. Shanafelt TD, Bradley KA, Wipf JE, Back AL. Burnout and self-reported patient care in an internal medicine residency program. Ann Intern Med 2002;136:358-367.

6. Shanafelt TD, Balch CM, Bechamps GJ, Russell T, Dyrbye L, Satele D, et al. Burnout and career satisfaction among American surgeons. Ann Surg 2009;250:463-471.

7. Shanafelt TD, Boone S, Tan L, Dyrbye LN, Sotile W, Satele D, et al. Burnout and satisfaction with work-life balance among US physicians relative to the general US population. Arch Intern Med 2012;172:1377-1385.

8. Shanafelt TD, Hasan O, Dyrbye LN, Sinsky C, Satele D, Sloan J, et al. Changes in burnout and satisfaction with work-life balance in physicians and the general US working population between 2011 and 2014. Mayo Clin Proc 2015;90:1600-1613.

9. Vijendren A, Yung M, Sanchez J. The ill surgeon: A review of common work-related health problems amongst UK surgeons. Langenbecks Arch Surg 2014;399:967-979.

10. Arora M, Diwan AD, Harris IA. Burnout in orthopaedic surgeons: A review. ANZ J Surg 2013;83:512-515.

11. Saleh KJ, Quick JC, Conaway M, Sime WE, Martin W, Hurwitz S, et al. The prevalence and severity of burnout 
among academic orthopaedic departmental leaders. J Bone Joint Surg Am 2007;89:896-903.

12. Saleh KJ, Quick JC, Sime WE, Novicoff WM, Einhorn TA. Recognizing and preventing burnout among orthopaedic leaders. Clin Orthop Relat Res 2009;467:558-565.

13. Sargent MC, Sotile W, Sotile MO, Rubash H, Barrack RL. Quality of life during orthopaedic training and academic practice, Part 1: Orthopaedic surgery residents and faculty. J Bone Joint Surg Am 2009;91:2395-2405.

14. Toker S, Melamed S, Berliner S, Zeltser D, Shapira I. Burnout and risk of coronary heart disease: A prospective study of 8838 employees. Psychosom Med 2012;74: 840-847.

15. Shanafelt TD, Balch CM, Dyrbye L, Bechamps G, Russell T, Satele D, et al. Special report: Suicidal ideation among American surgeons. Arch Surg 201 1;146:54-62.

16. Campbell DA Jr, Sonnad SS, Eckhauser FE, Campbell KK, Greenfield LJ. Burnout among American surgeons. Surgery 2001;130:696-702. discussion 702-705.

17. Shanafelt TD, Balch CM, Bechamps G, Russell T, Dyrbye L, Satele D, et al. Burnout and medical errors among American surgeons. Ann Surg 2010;251:995-1000.

18. Rosen IM, Gimotty PA, Shea JA, Bellini LM. Evolution of sleep quantity, sleep deprivation, mood disturbances, empathy, and burnout among interns. Acad Med 2006;81: 82-85.

19. Thomas NK. Resident burnout. JAMA 2004;292: 2880-2889.

20. Balch CM, Freischlag JA, Shanafelt TD. Stress and burnout among surgeons: Understanding and managing the syndrome and avoiding the adverse consequences. Arch Surg 2009;144:371-376.

21. Balch CM, Shanafelt T. Combating stress and burnout in surgical practice: A review. Adv Surg 2010;44:29-47.

22. Watson NF, Badr MS, Belenky G, Bliwise DL, Buxton OM, Buysse D, et al. Recommended amount of sleep for a healthy adult: A joint consensus statement of the American Academy of Sleep Medicine and Sleep Research Society. Sleep 2015;38:843-844.

23. Sargent MC, Sotile W, Sotile MO, Rubash H, Barrack RL. Stress and coping among orthopaedic surgery residents and faculty. J Bone Joint Surg Am 2004;86-A:1579-1586.

24. Philibert I, Friedmann P, Williams WT, Education AWGoRDHACfGM. New requirements for resident duty hours. JAMA 2002;288:1112-1114.

25. Nasca TJ, Day SH, Amis ES Jr, Force ADHT. The new recommendations on duty hours from the ACGME Task Force. N Engl J Med 2010;363:e3.

26. Bilimoria KY, Chung JW, Hedges LV, Dahlke AR, Love R, Cohen ME, et al. National Cluster-Randomized Trial of duty-hour flexibility in surgical training. $N$ Engl J Med 2016;374:713-727.

27. Fonseca P, Weysen T, Goelema MS, Most EIS, Radha M, Lunsingh Scheurleer C, et al. Validation of photoplethysmography-based sleep staging compared with polysomnography in healthy middle-aged adults. Sleep 2017;40:Zsx097.

28. Grunfeld E, Whelan TJ, Zitzelsberger L, Willan AR, Montesanto B, Evans WK. Cancer care workers in
Ontario: Prevalence of burnout, job stress and job satisfaction. CMAJ 2000;163:166-169.

29. Melamed S, Shirom A, Toker S, Berliner S, Shapira I. Burnout and risk of cardiovascular disease: Evidence, possible causal paths, and promising research directions. Psychol Bull 2006;132:327-353.

30. Pilkonis PA, Choi SW, Reise SP, Stover AM, Riley WT, Cella D, et al. Item banks for measuring emotional distress from the Patient-Reported Outcomes Measurement Information System (PROMIS): Depression, anxiety, and anger. Assessment $2011 ; 18: 263-283$.

31. Buysse DJ, Yu L, Moul DE, Germain A, Stover A, Dodds NE, et al. Development and validation of patientreported outcome measures for sleep disturbance and sleep-related impairments. Sleep 2010;33:781-792.

32. Volpp KG, Rosen AK, Rosenbaum PR, Romano PS, EvenShoshan O, Wang Y, et al. Mortality among hospitalized Medicare beneficiaries in the first 2 years following ACGME resident duty hour reform. JAMA 2007;298: 975-983.

33. Volpp KG, Rosen AK, Rosenbaum PR, Romano PS, EvenShoshan O, Canamucio A, et al. Mortality among patients in VA hospitals in the first 2 years following ACGME resident duty hour reform. JAMA 2007;298:984-992.

34. Rajaram R, Chung JW, Jones AT, Cohen ME, Dahlke AR, Ko CY, et al. Association of the 2011 ACGME resident duty hour reform with general surgery patient outcomes and with resident examination performance. JAMA 2014;312:2374-2384.

35. Rajaram R, Chung JW, Cohen ME, Dahlke AR, Yang AD, Meeks JJ, et al. Association of the 2011 ACGME resident duty hour reform with postoperative patient outcomes in surgical specialties. J Am Coll Sur 2015;221:748-757.

36. Browne JA, Cook C, Olson SA, Bolognesi MP. Resident duty-hour reform associated with increased morbidity following hip fracture. J Bone Joint Surg Am 2009;91: 2079-2085.

37. Ahmed N, Devitt KS, Keshet I, Spicer J, Imrie K, Feldman L, et al. A systematic review of the effects of resident duty hour restrictions in surgery: Impact on resident wellness, training, and patient outcomes. Ann Surg 2014;259:1041-1053.

38. Balch CM, Shanafelt TD, Dyrbye L, Sloan JA, Russell TR, Bechamps GJ, et al. Surgeon distress as calibrated by hours worked and nights on call. J Am Coll Surg 2010;211: 609-619.

39. Dahlke AR, Johnson JK, Greenberg CC, Love R, Kreutzer L, Hewitt DB, et al. Gender Differences in utilization of duty-hour regulations, aspects of burnout, and psychological well-being among general surgery residents in the United States. Ann Surg 2018;264:204-211.

40. Dyrbye LN, Shanafelt TD, Balch CM, Satele D, Sloan J, Freischlag J. Relationship between work-home conflicts and burnout among American surgeons: A comparison by sex. Arch Surg 2011;146:211-217.

41. Martini S, Arfken CL, Balon R. Comparison of burnout among medical residents before and after the implementation of work hours limits. Acad Psychiatry 2006;30: 352-355. 